\title{
Heterotopic Cesarean Scar Pregnancy in a Spontaneous Cycle
}

\author{
Chin-Jung Wang, MD, Yao-Lung Chang, MD, Hsing-Tse Yu, MD, Pei-Ju Wu, MD, \\ Shang-Gwo Horng, MD, Chia-Chang Tsai, MD \\ Department of Obstetrics and Gynecology, Chang Gung Memorial Hospital at Linkou, Tao-Yuan, Taiwan (Drs. Wang, Chang, Yu). \\ Department of Obstetrics and Gynecology, Chang Gung Memorial Hospital at Linkou and Chang Gung University of \\ Medicine, Tao-Yuan, Taiwan (Dr. Wang). \\ Department of Obstetrics and Gynecology, Chung Shan Medical University Hospital, Taichung, Taiwan (Dr. Wu). \\ Department of Obstetrics and Gynecology, Hsinchu Cathay General Hospital, Hsinchu, Taiwan (Dr. Horng). \\ Tsai's Women and Children Clinics, New Taipei, Taiwan (Dr. Tsai).
}

\begin{abstract}
Introduction: We report a viable cesarean scar pregnancy with a coexistent intrauterine pregnancy, managed successfully with operative hysteroscopy and suction curettage.

Case Description: A 32-year-old woman, with previous cesarean delivery, presented with vaginal bleeding and was referred to our hospital at 9 weeks' gestation for heterotopic pregnancy in a spontaneous cycle. An intrauterine pregnancy combined with a cesarean scar pregnancy was shown by ultrasonography. The patient underwent hysteroscopy-directed evacuation of the cesarean scar pregnancy and suction curettage with good hemostasis.
\end{abstract}

Discussion: Spontaneous heterotopic cesarean scar pregnancy is a rare but possible entity. The combination of hysteroscopy and suction curettage is an option for a minimally invasive procedure for heterotopic cesarean scar pregnancy.

Key Words: Cesarean scar, Heterotopic, Hysteroscopy, Pregnancy.

Citation Wang C-J, Chang Y-L, Yu H-T, Wu P-J, Horng S-G, Tsai C-C. Heterotopic cesarean scar pregnancy in a spontaneous cycle. CRSLS e2014.000993. DOI 10.4293/CRSLS.2014.000993

Copyright (C) 2014 SLS This is an open-access article distributed under the terms of the Creative Commons Attribution-Noncommercial-ShareAlike 3.0 Unported license, which permits unrestricted noncommercial use, distribution, and reproduction in any medium, provided the original author and source are credited.

Address correspondence to: Chin-Jung Wang, MD, 5 Fu-Hsin Street, Kwei-Shan, Tao-Yuan, Taiwan. Telephone: +886-3-328-8258; Fax: +886-3-328-8252; E-mail wang2260@gmail.com

\section{INTRODUCTION}

Heterotopic pregnancies (HPs) involving gestational tissue implanted in the endometrial cavity and extraendometrial cavity simultaneously are rare in the general population, with a range of occurrence estimated between 1 in 7963 and 1 in $30000 .^{1,2}$ With the increasing use of assisted reproductive technologies, the incidence of $\mathrm{HP}$ is estimated to be as high as $0.2 \%$ to $1 \% .^{3}$ According to a review of the published literature from 1994 to 2004, most of the HPs were located in the fallopian tube (72.5\%) and 3.8\% were located on cesarean scars. ${ }^{4}$ Heterotopic cesarean scar pregnancy (HCSP) is even more unusual in a spontaneous cycle.

Management of cesarean scar pregnancy (CSP) itself carries risk of uncontrollable bleeding, not to mention the salvage of the coexisting intrauterine pregnancy. In a previous report, we demonstrated successful conservative management of HCSP after in vitro fertilization-embryo transfer and preservation of intrauterine pregnancy. ${ }^{5}$ We report a case of HCSP in a spontaneous cycle, managed successfully by hysteroscopy-assisted suction curettage. In addition, a review of the published literature on spontaneous HCSPs diagnosed and treated from January 1992 to January 2012 is presented.

\section{CASE REPORT}

A 32-year-old Taiwanese woman, gravida 4, para 2, presented with vaginal bleeding. Her second infant was delivered by transverse lower-segment cesarean section 2 years earlier. The patient was referred to our hospital at 9 weeks' 


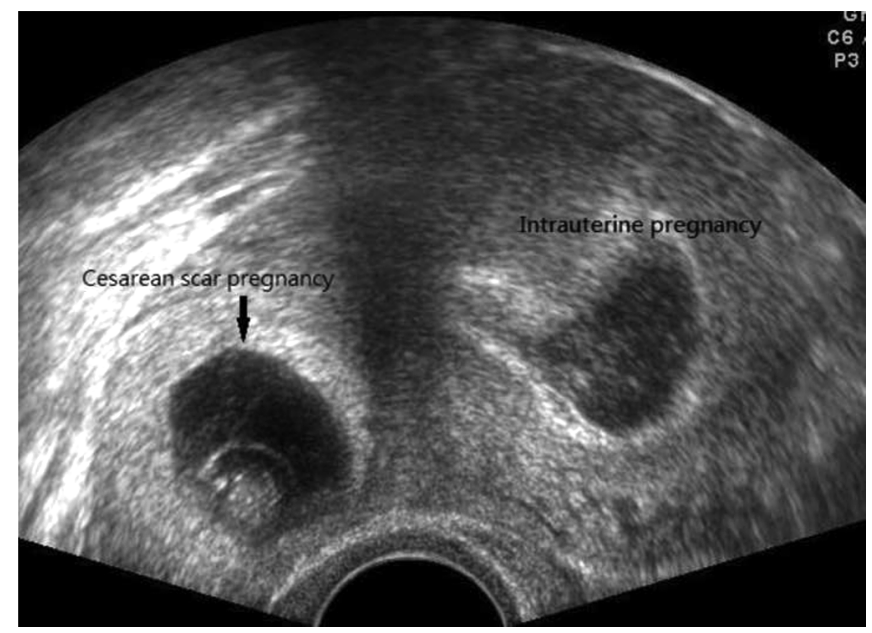

Figure 1. An ultrasonographic image shows a heterotopic twin pregnancy with one gestational sac located in the lower portion of the uterine cavity and the other within the cesarean section scar.

gestation for sonographic suspicion of a cervical pregnancy. Transvaginal sonography showed 2 gestational sacs, 1 located in the uterine lower portion without fetal cardiac activity and 1 located in the previous cesarean scar (Figure 1). The sac within the previous cesarean scar contained a yolk sac and a viable embryo measuring 8 weeks 2 days of gestation. Both ovaries appeared normal, and there were no adnexal masses or free fluid in the cul-de-sac. The plasma $\beta$ human chorionic gonadotropin level was $68500 \mathrm{mIU} / \mathrm{mL}$, and general physical examination findings were normal. A diagnosis of HCSP was highly suspected. The woman did not desire to continue the pregnancy. After counseling, the couple opted for conservative treatment with operative hysteroscopy and suction curettage.

Under general anesthesia without endotracheal intubation, the patient was placed in the dorsolithotomy position. After a speculum was placed inside the vagina, a tenaculum was applied to the cervix and gentle traction was exerted to align the uterus. The cervix was dilated by Hegar dilators to $12 \mathrm{~mm}$, and a continuous-flow 26F hysteroscopic resectoscope (Karl Storz, Tuttlingen, Germany) with a $90^{\circ}$ wire loop electrode was introduced under ultrasonographic control. Uterine distension was achieved with distilled water propelled by simple gravity. An Aspen Excalibur electrosurgical generator (Aspen Labs, Englewood, Colorado) was used at a setting of $80 \mathrm{~W}$ of cutting waveform current and $100 \mathrm{~W}$ of coagulation current.

The intervention began by identifying the implantation of the ectopic sac. The electric loop of the resectoscope was used to push the gestational sac, exposing the vessel bed of the implantation site. A coagulation current of $100 \mathrm{~W}$ was used for hemostasis. A placenta forceps was then used to pull out the gestational sacs under sonographic guidance, and suction curettage was used to clear the residual gestational tissue. Finally, a hysteroscopic rolling ball was used to stop the bleeding point. The operating time was 16 minutes. Vaginal bleeding was minimal at the end of the procedure. Postoperative ultrasonography showed a normal anteverted uterus with an intact endometrium and complete disappearance of the ectopic gestational sac. The patient had an unremarkable postoperative course and was discharged 3 hours after the procedure. A urine pregnancy test was negative on the 30-day postoperative visit, and normal echotexture of the uterus was noted. Menstruation resumed 4 days after this visit, and there has been no subsequent abnormal uterine bleeding within the 4-month follow-up period.

\section{DISCUSSION}

The exact incidence rate of HCSP is hard to estimate. A total of 19878 pregnancies (unwanted pregnancies not included) were delivered and 846 ectopic pregnancies received treatment in our department from January 2007 to December 2011. Among these cases, 3 patients were diagnosed with HCSP and 1 of these 3 patients conceived spontaneously. Therefore the incidence rate of HCSP was at least $0.005 \%$ (1 of 20724 ) at our hospital (a 3 900-bed, large medical center).

We performed a review of the published literature from January 1992 to January 2012 using the PubMed database. The medical terms used were heterotopic pregnancy, cesarean scar pregnancy, and cervical pregnancy. Available data on the patients' clinical characteristics, treatment, and outcome are summarized in Table 1. Our previous case and current case were included.

Ultrasonography is the primary imaging technique for diagnosing HCSP. The sonographic criteria for HCSP on a sagittal view of the uterus are as follows: a gestational sac with or without cardiac activity located anteriorly at the level of the internal os, equivalent to a prior lower uterine segment with a cesarean section scar; a bulging gestational sac with a diminished myometrial layer between the bladder and the sac; and the other gestational sac located in the normal endometrial cavity. 5,6 The gestational sac in a spontaneous abortion in progress is avascular; therefore Doppler ultrasonography can provide more detail on the trophoblastic circulation of implantation to make a prompt differential diagnosis. ${ }^{7}$

At present, there is no standard treatment protocol for HCSP. Feticide with potassium chloride $(\mathrm{KCl})$ under trans- 


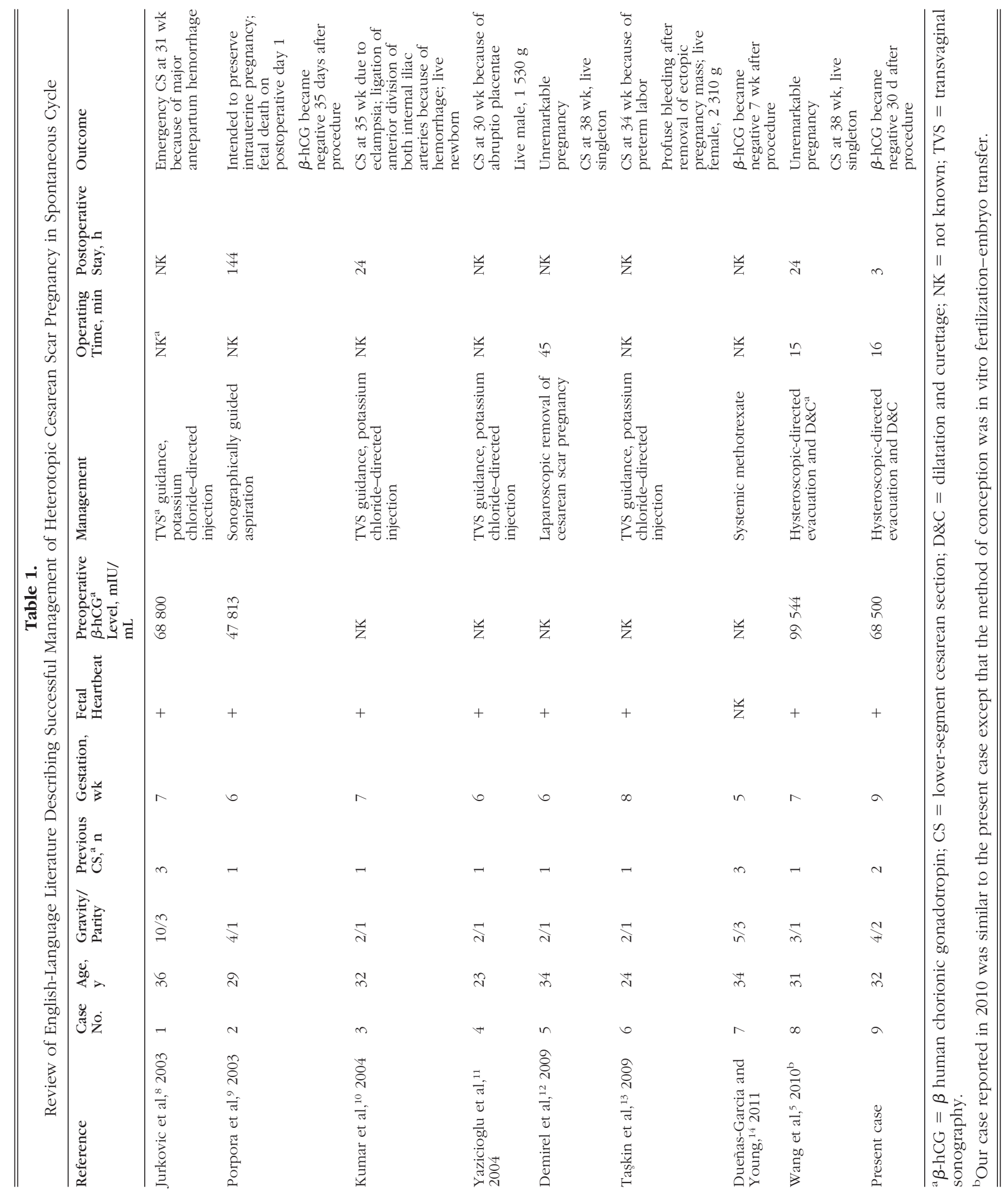


vaginal sonographic guidance, systemic methotrexate, embryo aspiration, hysteroscopy, and laparoscopic management of CSP have emerged as minimally invasive procedures. ${ }^{5,8-14}$ The pregnant courses of patients who underwent fetal reduction with gestational tissue in situ were not uneventful. All babies were born prematurely because of various causes (severe antepartum hemorrhage, eclampsia, abruptio placentae, or preterm labor). Immediate hemorrhage was even encountered in 2 pregnancies, and bilateral hypogastric artery ligation was necessary to stop the bleeding in 1 woman for preservation of the uterus. In contrast, surgical removal of an HCSP carried an uneventful prenatal course and the pregnant woman delivered a live birth at term (Table 1).

Selective reduction of CSPs with $\mathrm{KCl}$ under ultrasonographic guidance is the most common type of medical treatment. $8,10,11,13$ The potential teratogenic effect of methotrexate forbids its use in the preservation of intrauterine gestation in HCSPs. Direct embryo aspiration may be an alternative but might affect the intrauterine pregnancy. ${ }^{\text {? }}$

Removal of the ectopic mass and repair of the uterine defect by laparoscopy or laparotomy was reported to be a safe technique.,12 A disadvantage of this transabdominal approach was that the healthy uterine tissue was not easy to dissect and identify because of the overlying bladder. We previously reported hysteroscopic management of a firsttrimester CSP and an HCSP with preservation of intrauterine gestation and delivery at term.5,6 When the patient has no desire to continue the pregnancy, the management of HCSP is similar to that of a single CSP; for preservation of the intrauterine gestation, hysteroscopic manipulation should be reduced as minimally as possible because the intrauterine embryo might be disturbed by the upward force of the distended medium during hysteroscopy. The hysteroscope should not be placed beyond the endocervical canal and should be withdrawn immediately and replaced by placenta forceps and suction curettage to remove the gestational tissues under sonographic guidance after identifying the location of the CSP and coagulating the vessel beds of the implantation site.

\section{CONCLUSION}

HCSP is a rare form of ectopic pregnancy, and it mainly occurs after in vitro fertilization-embryo transfer. In our patient this condition occurred spontaneously. The preservation of the normally implanted embryo in HCSP remains more challenging. Although fetal reduction with $\mathrm{KCl}$ is frequently adopted, on the basis of the reported cases and our experience, we suggest that hysteroscopic management is another reasonable option but is not necessarily better and requires more data. Ultrasonography can provide a reliable and early diagnosis of HCSP. Early diagnosis is warranted to preserve fertility and avoid maternal morbidity.

\section{References:}

1. Reece EA, Petrie RH, Sirmans MF, Finster M, Todd WD. Combined intrauterine and extrauterine gestations: a review. Am J Obstet Gynecol. 1983;146:323-330.

2. Chin HY, Chen FP, Wang CJ, Shui LT, Liu YH, Soong YK. Heterotopic pregnancy after in vitro fertilization-embryo transfer. Int J Gynaecol Obstet. 2004;86:411-416.

3. Dor J, Seidman DS, Levran D, Ben-Rafael Z, Ben-Shlomo I, Mashiach $S$. The incidence of combined intrauterine and extrauterine pregnancy after in vitro fertilization and embryo transfer. Fertil Steril. 1991;55:833-834.

4. Barrenetxea G, Barinaga-Rementeria L, Lopez de Larruzea A, Agirregoikoa JA, Mandiola M, Carbonero K. Heterotopic pregnancy: two cases and a comparative review. Fertil Steril. 2007;87:417.e9-417.e15.

5. Wang CJ, Tsai F, Chen C, Chao A. Hysteroscopic management of heterotopic cesarean scar pregnancy. Fertil Steril. 2010; 94:1529.e15-1529.e18.

6. Wang CJ, Chao AS, Yuen LT, Wang CW, Soong YK, Lee CL. Endoscopic management of cesarean scar pregnancy. Fertil Steril. 2006;85:494.e1-494.e4.

7. Rotas MA, Haberman S, Levgur M. Cesarean scar ectopic pregnancies: etiology, diagnosis, and management. Obstet Gynecol. 2006;107:1373-1381.

8. Jurkovic D, Hillaby K, Woelfer B, Lawrence A, Salim R, Elson CJ. First-trimester diagnosis and management of pregnancies implanted into the lower uterine segment Cesarean section scar. Ultrasound Obstet Gynecol. 2003;21:220-227.

9. Porpora MG, D'Elia C, Bellavia M, Pultrone DC, Cosmi EV. Heterotopic cervical pregnancy: a case report. Acta Obstet Gynecol Scand. 2003;82:1058-1059.

10. Kumar S, Vimala N, Dadhwal V, Mittal S. Heterotopic cervical and intrauterine pregnancy in a spontaneous cycle. Eur J Obstet Gynecol Reprod Biol. 2004;112:217-220.

11. Yazicioglu HF, Turgut S, Madazli R, Aygun M, Cebi Z, Sonmez S. An unusual case of heterotopic twin pregnancy managed successfully with selective feticide. Ultrasound Obstet Gynecol. 2004;23:626-627.

12. Demirel LC, Bodur H, Selam B, Lembet A, Ergin T. Laparoscopic management of heterotopic cesarean scar pregnancy with preservation of intrauterine gestation and delivery at term: case report. Fertil Steril. 2009;91:1293.e5-1293.e7.

13. Taşkin S, Taşkin EA, Ciftçi TT. Heterotopic cesarean scar pregnancy: how should it be managed? Obstet Gynecol Surv. 2009;64:690695.

14. Dueñas-Garcia OF, Young C. Heterotopic cesarean scar pregnancy associated with a levonorgestrel-releasing intrauterine device. Int J Gynaecol Obstet. 2011;114:153-154. 